\title{
Editor Welcome
}

\section{Claudio L. Battaglini ${ }^{1}$}

Published online: 15 October 2020

(c) Beijing Sport University 2020

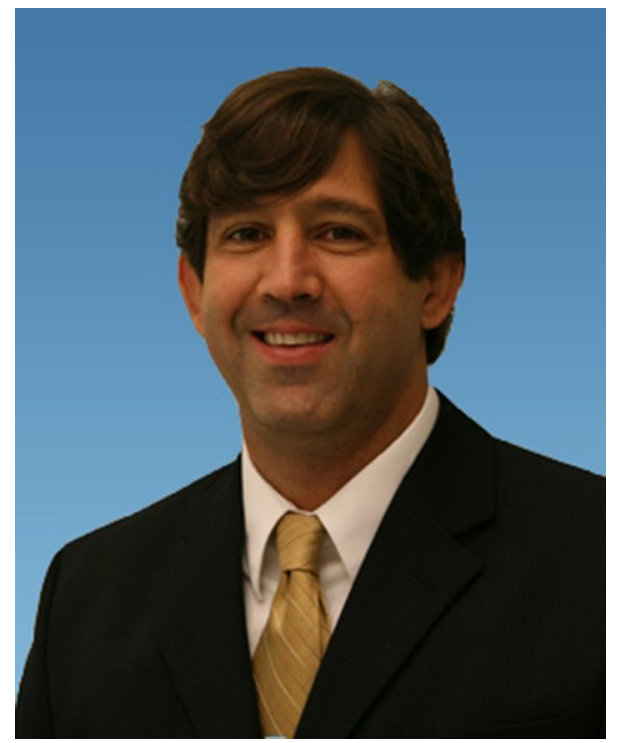

This special issue of the Journal of Science in Sport and Exercise is devoted to scientific inquiry related to clinical exercise and sports medicine. Each article is authored by top researchers in the clinical exercise physiology and sports medicine community.

In this special issue, the fairly new, exciting, and rapidly growing area of exercise oncology is highlighted with three reviews and one original research article from some of the best laboratories in the US, Germany, and Brazil. These articles provide thought provoking questions and the latest insights in the area of exercise oncology including current evidence and recommendations for future research.

Claudio L. Battaglini

claudio@email.unc.edu

1 Department of Exercise and Sport Science, Lineberger Comprehensive Cancer Center, University of North Carolina at Chapel Hill, Chapel Hill, USA
There are three articles ( 2 reviews and 1 original research article) detailing the prescription of exercise for adults with diabetes in Japan, the impact of sleep in children's early development of central arterial stiffness and a review article on the molecular and cellular aspects of sarcopenia in elderly. All three articles address the critical role of exercise in preventing and/or attenuating further health decrements in children and adults at risk or already experiencing these commonly diagnosed chronic conditions.

Finally, two articles exploring the ever growing issue associated with orthopedic injuries from prevention to treatment of advanced injury perspectives conclude this special issue. The first article provides the latest information on the management of early risk for the development of posttraumatic osteoarthritis followed by knee injury and the second one a longitudinal analysis of movement coordination before and after knee injury conducted with the goal of identifying movement patterns that can be potentially corrected with specific exercise to minimize injury risk.

We believe that these nine articles are indicative of the breadth, depth, and excitement of the ever growing clinical exercise and sports medicine topics that are representative of some of the most important topics currently affecting today's world. We hope you enjoy reading them as much as we did.

Qhimaio Batteg Din

Claudio L. Battaglini Phd, FACSM

Guest Editor 